\title{
Virtualización de las asignaturas de estudios generales en la formación universitaria. Propuesta de concepción teórico- metodológica para su aplicación
}

\section{Virtualization of general studies subjects in university education. Proposal of a theoretical-methodological conception for its application}

\author{
José Alberto Medina Crespo 1 https://orcid.org/0000-0002-7052-5668 \\ ${ }^{1}$ Universidad Católica de Santiago de Guayaquil, Guayaquil, Ecuador \\ jose.medina@cu.ucsg.edu.ec
}

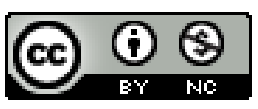

Esta obra está bajo una licencia internacional Creative Commons Atribución-NoComercial 4.0.

$\begin{array}{ll}\text { Enviado: } & 2021 / 07 / 06 \\ \text { Aceptado: } & 2021 / 09 / 28 \\ \text { Publicado: } & 2021 / 11 / 30\end{array}$

\section{Resumen}

Los estudios generales en la formación universitaria al ser de tronco común en todas las carreras han acarreado problemas de espacio para cumplir con su oferta. Estas asignaturas proporcionan conocimientos y habilidades que complementan la formación profesional. La virtualización permite integrar o reemplazar, según sea el caso, la presencialidad en los procesos de enseñanza-aprendizaje, lo que favorece al ahorro de recursos y la adaptación en espacio-tiempo de las necesidades de los estudiantes y docentes conforme a la disponibilidad de herramientas tecnológicas que en la actualidad forman parte del proceso educativo. Este informe desarrolla una concepción teórico-metodológica con dimensiones y componentes que orientan el proceso de virtualización educativa para los estudios generales en la formación universitaria, como opción para la optimización de recursos y generación de nuevos espacios de aprendizaje basados en dimensiones con dos componentes: el teórico y el metodológico, en el cual se precisan las orientaciones para el desarrollo de la concepción. En el componente teórico se establecen las bases conceptuales de la propuesta mientras que en el componente metodológico se presentan las etapas del componente y sus respectivas acciones para su implementación en las asignaturas de estudios generales en la formación universitaria.

Palabras clave: virtualización, educación superior, estudios generales, universidad, formación general universitaria.

Sumario: Introducción, Materiales y Métodos, Resultados y Discusión y Conclusiones.

Como citar: Medina, J. (2021). Virtualización de las asignaturas de estudios generales en la formación universitaria. Propuesta de concepción teórico-metodológica para su aplicación. Revista Tecnológica Espol, 33(2), 122-133. http://www.rte.espol.edu.ec/index.php/tecnologica/article/view/837 


\begin{abstract}
The general studies in university education, being a common core in all careers, have caused problems of space to comply with the offer of these courses. These courses provide knowledge and skills that complement professional training. Virtualization makes it possible to complement or replace face-to-face attendance in the teaching-learning processes, thus saving resources and adapting space and time to the needs of students and teachers according to the availability of technological tools that are currently part of the educational process. A theoretical-methodological conception was developed with dimensions and components that guide the process of educational virtualization for general studies in university education, as an option for the optimization of resources and generation of new learning spaces based on dimensions with two components: the theoretical and the methodological in which the orientations for the development of the conception are specified. The theoretical component establishes the conceptual bases of the proposal, while the methodological component presents the stages of the component and their respective actions for its implementation in the general studies subjects in university education.
\end{abstract}

Keywords: virtualization, higher education, general studies, university, general university training.

\title{
Introducción
}

El estudio general es un elemento que ha marcado la formación profesional en todos sus ámbitos. Para citar un ejemplo, la formación comunicativa no es solo ese medio para transmitir elementos abstractos de lenguaje sino también para transmitir capacidades que permitan el desenvolvimiento de un profesional de diferentes áreas del conocimiento y es por lo que se aborda como un elemento importante dentro formación universitaria.

En aproximación a lo que se menciona, el impacto de las Tecnologías de la Información y Comunicación en la actualidad juega un rol fundamental en el desarrollo de la sociedad en todas sus dimensiones. Es evidente que la tecnología, favorece a la educación ya que no solo permite desarrollar habilidades que hoy resultan necesarias, sino que proporciona herramientas a las instituciones, especialmente a la Educación Superior, para la mejora continua del proceso de enseñanza y aprendizaje (Medina \& Rea, 2017).

Tal es el caso de la importancia de la tecnología que la implicación de la internet y el desarrollo de plataformas virtuales se han integrado a procesos que van desde la planificación y evaluación académica hasta la eficiencia en la utilización de recursos, lo que genera mayor rentabilidad y un mejor servicio en lo que respecta a la disponibilidad (Buhalis \& Deimezi, 2003).

Dada la importancia del tema tecnológico en la Educación Superior, la Conferencia Mundial Sobre la Educación Superior en París (2009), precisa la importancia de las tecnologías como un mecanismo para el desarrollo sostenido de la educación. También de su renovación para enfrentar los retos que se generan ante nuevos escenarios sociales como el acceso al conocimiento y la eliminación de barreras que impidan la formación profesional (UNESCO, 2009).

De acuerdo con la Declaración de Quindao, propiciado por la Organización de las Naciones Unidas para la Educación y Cultura (UNESCO) en el año 2015, se desarrolla el Foro Mundial sobre TIC y Educación 2030 bajo el lema "aprovechar las oportunidades digitales, liderar la transformación de la educación", con la premisa de que la educación es fundamental para el desarrollo sostenible de las sociedades (UNESCO, 2019). 
De acuerdo con el Informe Horizon (EDUCASE, 2019) sobre tendencias en Tecnología Educativa en su edición 2019, las tecnologías se desarrollan de forma acelerada y son adoptadas de manera inmediata por la educación superior. Cabe destacar que el informe consolida las opiniones e informaciones de expertos internacionales en el cual destacan aspectos tales como el rediseño de los espacios de aprendizaje y los diseños de espacios combinados mixtos.

A partir de lo expuesto en este informe, es importante destacar que el diseño de los espacios de aprendizaje debe tener como centro a los estudiantes con una naturaleza colaborativa, pero con escenarios que propicien el proceso formativo desde el uso de herramientas digitales hasta la facilidad para el acceso a ellas. En concordancia con lo expuesto, la implementación de las TIC en la educación superior gana considerable impulso en los últimos años. El diseño de estos espacios facilita el aprendizaje activo y colaborativo. Requiere, por lo tanto, de inversión y planificación estratégica, para construir o renovar esos ambientes donde se lleva a cabo el proceso de enseñanza y aprendizaje.

\section{Materiales y Métodos}

El alcance de este estudio es descriptivo experimental con un enfoque metodológico mixto (Henández, Fernández, \& Baptista, 2014). De esta manera, como desarrollo de una metodología general en el proceso científico, se recurrió al empleo de otros métodos, instrumentos y técnicas que posibilitaron un análisis del objeto de la investigación.

Entre los métodos teóricos empleados se precisan los siguientes:

Histórico - lógico, para el estudio de la virtualización en la Educación Superior y su desarrollo a finales del siglo XX y el XXI en orden histórico (orígenes) y cronológico (desarrollo), al revelarse sus particularidades, contradicciones y las marcadas tendencias en el proceso formativo universitario actual como fundamento de la estrategia teórica-metodológica propuesta.

Analítico - Sintético, que se empleó en el procesamiento de la información teórica acerca de la virtualización en la educación superior y de los datos obtenidos de la aplicación de instrumentos para el diseño del diagnóstico y la valoración de la concepción teórica metodológica de virtualización de las asignaturas de estudios generales en la carrera de Turismo de la Universidad Católica de Santiago de Guayaquil (UCSG).

A continuación, se precisan los métodos empíricos empleados en el estudio:

Estudio documental que contribuyó en la elaboración de la fundamentación teóricometodológica de la propuesta, mediante la revisión bibliográfica y el análisis de la contextualización de la virtualización en la educación superior de Ecuador, en lo que respecta a los órganos rectores de la educación superior nacional y la propia UCSG, en las dimensiones jurídicas, pedagógicas, tecnológicas y organizacional.

Medición, se utilizó un cuestionario para obtener información sobre la base de los criterios de docentes, directivos, empleadores, estudiantes recién graduados y expertos durante la aplicación de la técnica de grupos focales, lo que permitió corroborar la concepción teórica metodológica en términos de pertinencia y factibilidad.

Observación científica: a partir de una guía de observación se valoró el estado actual del problema y la recepción de información en el propio desarrollo de actividades donde se empleen las TIC. 
Criterios de expertos y Técnica de grupos focales, se emplearon para la obtención de criterios durante la valoración cualitativa de la concepción teórica-metodológica, lo cual permitió corroborarla en términos de pertinencia y factibilidad.

Modelación y experimentación, mediante la presentación gráfica de la concepción metodológica y su aplicación a modo de prueba para las respectivas conclusiones del investigador.

Métodos matemáticos y estadísticos, se emplearon en la cuantificación y procesamiento de la información obtenida a partir de operaciones porcentuales, media y empleo de base de datos.

Para el desarrollo de esta investigación, la población estuvo representada por coordinadores, docentes y estudiantes que conformaron la totalidad de estudiantes inscritos en la carrera de Turismo de la UCSG en el Semestre B (octubre - febrero 2018). La muestra fue determinada mediante la técnica de muestreo aleatorio simple.

Para el proceso de diagnóstico, se aplicaron instrumentos como la encuesta, entrevista y la observación científica para la determinación de resultados en función de la participación de estudiantes de la carrera de Turismo, Directivos y Docentes referente a la virtualización del conjunto de asignaturas denominadas Estudios Generales, las cuales son: Idioma Español, Introducción al Pensamiento Crítico, Humanismo Integral y Estudios Contemporáneos. Esta última se tomó para la modelación de la concepción teórico-metodológica.

Para diagnosticar la situación actual de la virtualización de las asignaturas de estudios generales en la Carrera de Turismo, se aplicó el cálculo de la muestra finita debido a que se conoce con precisión la cantidad de la población, para la determinación de la muestra y empleo de la encuesta (Rodríguez L. , 2007). En el semestre B 2018, la carrera registró una población total de 420 estudiantes en la modalidad presencial.

Realizado el cálculo, se determinó que la cantidad de la muestra fue de 201 estudiantes. Dentro de la muestra se tomó a estudiantes de los niveles básico y básico específico, niveles donde se encuentran las asignaturas de estudios generales y se utilizó la técnica de muestreo aleatorio simple, de los cuales el primer grupo corresponde a un total de 85 estudiantes $(71 \%$ mujeres, $29 \%$ hombres) y al segundo grupo un total de 116 estudiantes (60\% mujeres, $40 \%$ hombres).

Para los datos cuantitativos se utilizó a la población total de docentes de la carrera, quienes, en la fecha de aplicación del estudio, tenían más de cinco años de experiencia en docencia universitaria. Constaba de la siguiente forma: 4 docentes Titulares Auxiliares (25\%), 10 docentes ocasionales a tiempo completo (38\%), 5 coordinadores de área (31\%) y la directora de carrera (6\%). De la población docente de la carrera, el $81 \%$ tenía solo título de maestría y el 19\% la maestría y cursaban estudios doctorales. En referencia a su afinidad, el 50\% fueron graduados en Turismo, 38\% en Ciencias Administrativas y Empresariales, y el 12\% en Lengua Inglesa.

En el resultado de las encuestas aplicadas a los estudiantes se halló que el 39\% de los encuestados manifestaron que el problema fue el cruce de horarios, ya que las bandas horarias se realizaban en función de las asignaturas específicas de las carreras sin tomar en cuenta las de estudios generales. El 31\% de los encuestados mencionaron que los horarios no fueron 
adecuados debido a que se implementaban en horas laborables lo que complicaba a quienes trabajaban.

Un $26 \%$ en cambio señalaron que el problema fue la disponibilidad de cupos por la alta demanda ya que son asignaturas que se integran en todas las carreras de la UCSG y finalmente, un $4 \%$ indicó no haber tenido problemas.

El Reglamento de Régimen Académico del Ecuador precisa que las programaciones académicas pueden optar por la modalidad blended learning que consiste en asistir a clases y tener ciertas horas de trabajo en plataforma lo que ha sido una alternativa socializada por la carrera en referencia a este documento. En este sentido se observó que un $70 \%$ de los encuestados mencionaron que, conocen lo estipulado en el Reglamento, mientras que un $30 \%$ indicó no conocerlo.

Se generó una expectativa para la solución de los inconvenientes generados al momento del registro, basado en la alternativa brindada por el régimen de educación superior ecuatoriano para que los estudiantes puedan realizar una modalidad diferente a la que están registrados, sin que afecte a sus créditos, siempre y cuando esta modalidad sea dada por una universidad acreditada.

Al consultarse sobre si la virtualización de las asignaturas de estudios generales mejoraría la disponibilidad de los cursos, el 60\% manifestó que definitivamente esto mejorará la disponibilidad de estas asignaturas, y sus problemas para el registro. Un 30\% precisó en un nivel de confianza no tan alto que probablemente si lo mejoraría y en último lugar un $10 \%$ estimó que de ninguna manera lo mejorará y que más bien será igual.

Con lo mencionado anteriormente, se hace referencia al uso de la plataforma Moodle UCSG como herramienta para la virtualización de las asignaturas de estudios generales en donde el $92 \%$ de los encuestados consideraron tomar las asignaturas de estudios generales en línea, mientras que el $7 \%$ indicó que no y el 1\% le pareció indiferente el cuestionamiento. Este dato aportó con el seguimiento del hilo conductor de la encuesta la que va tomando forma de propuesta a la expectativa generada en los encuestados con respecto a la virtualización de las asignaturas de estudios generales en la carrera de Turismo de la UCSG.

La característica del aprendizaje en línea se fundamenta en la autonomía del estudiante resultando que un 31\% indicaron que con seguridad se sienten muy preparados para asumir el aprendizaje autónomo en las asignaturas de estudios generales; mientras que un 64\% representando la mayoría señalaron sentirse preparados, es decir, en un nivel menor a los anteriores. Finalmente, un 5\% de los encuestados manifestaron sentirse poco y nada preparados lo que se puede establecer que hay un escenario alentador en la implementación de un componente de formación de manera autónoma.

Uno de los aspectos que se establecen en los ambientes virtuales de aprendizaje, es la dinámica comunicacional determinada por las diferentes herramientas que brinda la plataforma. En este aspecto, se consultó que sin la comunicación entre docentes y estudiantes en las asignaturas de estudios generales sería más dinámica que en aula presencial, se obtuvo que un $80 \%$ piensa que sí, mientras que el $16 \%$ manifestó que no y un $4 \%$ les resulta indiferente. En esta pregunta se estructuró también un componente abierto, solicitando fundamentación de la respuesta en la cual la mayoría de los encuestados respondieron que, en la virtualidad, se puede ser más directo, espontáneo y sin temor de participar en actividades comunicacionales. 
Las impresiones de los estudiantes acerca de los docentes y el manejo de la plataforma fueron primordiales para las acciones propuestas en esta investigación. El 15\% consideraron alto mientras que un $38 \%$ suficiente y un $41 \%$ medio, lo que mostró un escenario positivo en cuanto a esta impresión. En lo que corresponde a nivel "bajo" solo 6\% de los encuestados lo consideraron así.

Presentada la propuesta de investigación, se consultó sobre la implementación de la virtualización de las asignaturas de estudios generales en la carrera de Turismo, dando un 93\% de aceptación favorable de los encuestados versus una cantidad de $5 \%$ y $2 \%$ que lo consideraron poco viable y nada viable respectivamente. Este resultado fue importante ya que en el desarrollo del cuestionario se condujo a la presentación de una propuesta la cual, por parte de los encuestados, la encuentran muy viable.

Ya en el cierre del cuestionario aplicado a los estudiantes, y con el desarrollo del hilo de las dimensiones de la concepción teórico-metodológica para la virtualización de las asignaturas de estudios generales en la carrera de Turismo, el 95\% de los encuestados indicaron su deseo de elegir estas asignaturas en la modalidad virtual. En el componente abierto de la pregunta en la cual se solicitó que se fundamente la respuesta, la mayoría de los encuestados indicaron que les resulta práctico y muy adecuada la alternativa para solucionar el problema de los horarios y que les parece muy atractiva esta modalidad de estudio combinada.

\section{Concepción Teórico Metodológica para la virtualización de estudios generales en la formación universitaria}

Con los resultados expuestos, se presenta una concepción Teórico-Metodológica para la virtualización de las asignaturas de estudios generales en la formación universitaria con una configuración que integra a los participantes de estos entornos, centrada en las necesidades del estudiante en un ambiente virtual. En esta concepción, el docente utiliza recursos y herramientas que se acoplan a la configuración del ambiente virtual y que permite el proceso de enseñanza aprendizaje en la ubicuidad (Barreto \& Iriarte, 2017).

Fainholc (2016) y Fernández (2019) expresan que son un soporte al proceso de enseñanza-aprendizaje para las modalidades existentes y en sus diferentes combinaciones, como el blended learning y el e-learning. Un buen diseño de un entorno virtual de enseñanza - aprendizaje (EVEA) evidencia objetivos claros y precisos en el proceso formativo y guarda estricta relación con los contenidos, indistintamente de la modalidad de estudio en una interfaz amigable que motive la interacción de los sujetos en formación y la elaboración de herramientas dinámicas en el contexto pedagógico-tecnológico para el desarrollo de habilidades y destrezas.

La concepción teórico-metodológica se basa en cinco dimensiones, las cuales se interrelacionan e interactúan entre sí con un enfoque integrador para tener un campo específico. Contiene dos componentes, los cuales el componente teórico de la concepción se fundamenta a partir de los referentes de tecnología educativa y las teorías asumidas por investigaciones que caracterizan los procesos formativos actuales y logran una adecuada virtualización de la educación superior. Y el componente metodológico se refiere a las fases, momentos y pasos concebidos para desplegar la concepción teórico-metodológica con etapas definidas. En la Figura 1 se detallan las etapas que son: Etapa Diagnóstica; Etapa de Planificación, Etapa Proyectiva; Etapa Desarrolladora, Etapa Evaluativa y Etapa Valorativa. 


\section{Figura 1}

Etapas del componente metodológico de la concepción propuesta

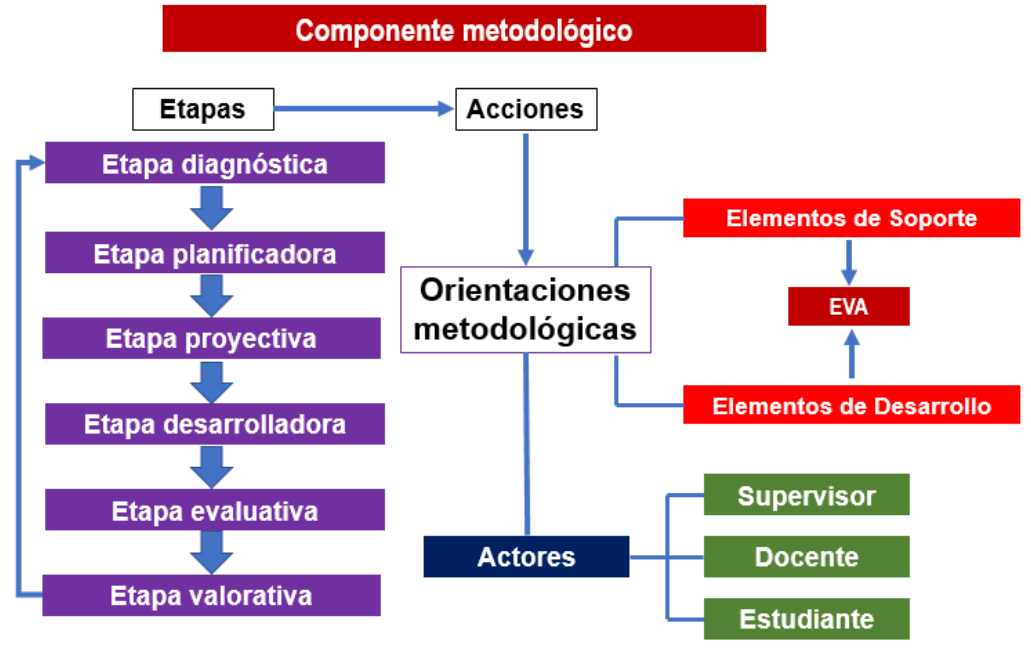

La Etapa Diagnóstica se caracteriza por conocer científicamente el estado real del objeto para determinar sus contradicciones e insuficiencias, evidencia el problema. Una premisa básica para lograr claridad de lo que se debe hacer con los estudiantes o grupo es la existencia de las etapas diagnósticas inicial, parciales y final. La Etapa de Planificación está dirigida a la determinación de cómo se desarrollará la tecnología educativa a emplear. Se tiene en cuenta el establecimiento del contexto y ubicación de las problemáticas a resolver, ideas y puntos de partida. Se caracteriza por poner en conocimiento de directivos, funcionarios y actores ejecutantes principales de cada uno de los años en la carrera, lo referente a concepción propuesta, y en consecuencia se planifiquen las acciones a tener en cuenta para la virtualización en dicha materia de la carrera en particular y del conjunto de acciones en los diferentes niveles de dirección y organizativos-metodológicos en general.

La Etapa Proyectiva responde al diseño de las líneas de acción resultantes del proceso de planificación, dirigidas a la definición de la guía para las acciones, a la ejecución de estas en su efecto. Establece la definición de metas u objetivos a corto y mediano plazo que permiten la transformación del proceso de enseñanza-aprendizaje desde su estado real hasta el estado deseado en función de la mediación de la tecnología educativa dispuesta, esto permite establecer las direcciones para el logro de dichas metas, y debe ser flexible y contextualizada. La Etapa Desarrolladora tiene como esencia la ejecución y desarrollo de las acciones y actividades planificadas desde la perspectiva del empleo directo de las tecnologías seleccionadas en el proceso de enseñanza-aprendizaje de las asignaturas de estudios generales de la carrera de turismo.

La Etapa Evaluativa se despliega en todas las etapas, pero en específico está presente en este apartado. Se expresa de manera gradual el tránsito del estado real al estado deseado. Se establecen formas de evaluación de acuerdo con las necesidades de aprendizaje de los estudiantes y de lo que plantean los objetivos a lograr por parte del currículo, así como también la evaluación del proceso formativo. La Etapa Valorativa tiene como centro de efecto expresar los logros y/o obstáculos sucedidos a lo largo del desarrollo de las etapas, sobre la base de la aproximación lograda desde el estado inicial al estado deseado.

Como la etapa anterior, también tiene una estrecha relación con las demás, ya que se van realizando en cada una de ellas valoraciones que al final se tienen en cuenta a modo de retroalimentación, con vistas al perfeccionamiento de la concepción en su totalidad. 
En lo referente a las acciones del componente metodológico, las acciones que se plantean en esta etapa son las siguientes:

$>$ Establecer un cronograma de cursos de capacitación a docentes y estudiantes en el manejo de las herramientas que se integran en esta concepción.

$>$ Diseñar de los cursos de manejo de herramientas tecnológicas y elaboración del Diseño Instruccional con el apoyo del Centro de Innovación Educativa y Desarrollo Docente de la UCSG.

$>$ Difusión de los cursos mediante el departamento de Comunicación y Marketing a estudiantes y docentes de la carrera de Turismo.

$>$ Comenzar el montaje de la plataforma con los recursos tecnológicos y educativos para la ejecución de las asignaturas de Estudios Generales en modalidad virtual.

La Concepción Teórico-Metodológica presentada en la Figura 2, constituye un abordaje para la utilización de los medios tecnológicos en el proceso de desarrollo integral del estudiante universitario. Se concibe con el propósito de lograr objetivos relacionados con conocimientos, habilidades y valores comunicativos en los modos de actuación profesional, que son clave en la formación superior y que no siempre es posible lograrlos con la debida profundidad desde la óptica de una sola disciplina o asignatura.

Se define y se diseña la concepción como una herramienta para el incremento de la pertinencia y la calidad en el proceso de formación general del futuro profesional universitario, lo cual, permite organizar las asignaturas de estudios generales en un entorno virtual que genera el desarrollo de habilidades y competencias tecnológicas en su formación.

El funcionamiento de la concepción persigue un alejamiento de la improvisación y lo circunstancial. También, permite insertar las actividades que contemplan fundamentos teóricos, técnicos-metodológicos, criterios de diseño, sistematización, controles $\mathrm{y}$ retroalimentación, para alcanzar un resultado que satisfaga la necesidad y creatividad de su elaboración, y orientar estratégicamente la actividad formativa en el proceso de enseñanza y aprendizaje. A continuación, se representa de manera gráfica la concepción teóricometodológica con todos los elementos que lo componen.

\section{Figura 2}

Representación gráfica de la concepción teórico-metodológico para la virtualización de las asignaturas de estudios generales en la formación universitaria

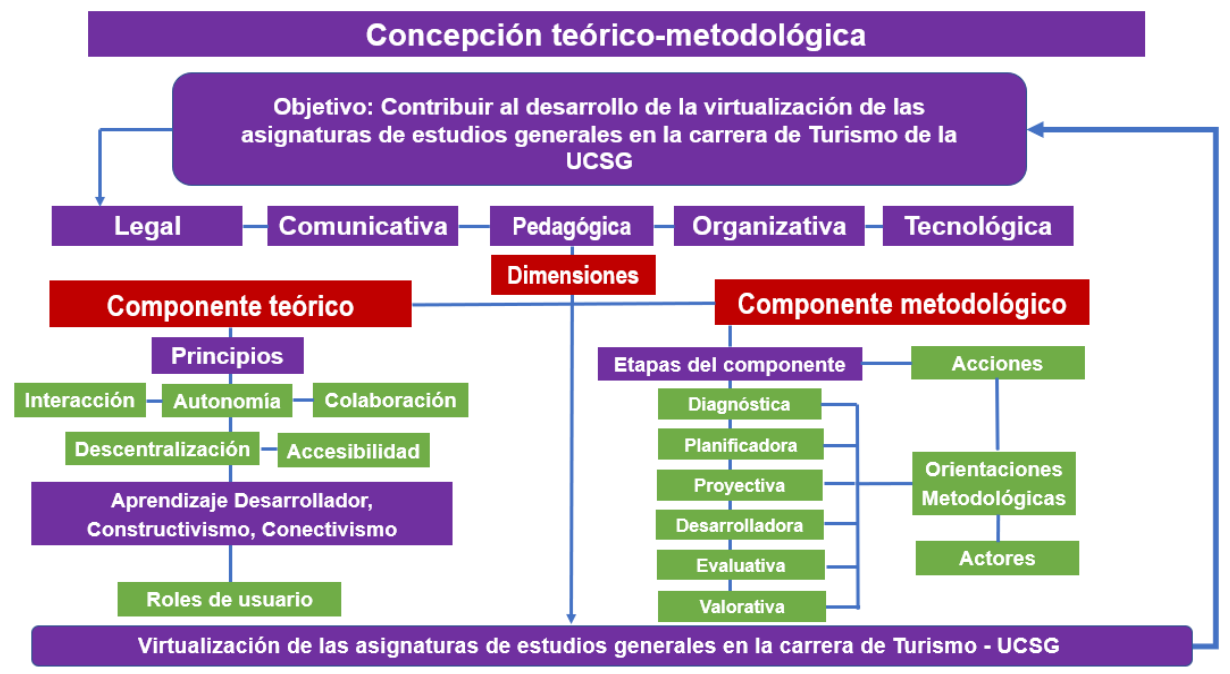




\section{Resultados y Discusión}

Aplicada la modelación de la concepción teórico-metodológica, se aplica una encuesta a los estudiantes participantes de la modelación del aula de prueba. La plataforma se estructura en una sección de presentación de la asignatura, organización y recursos para el seguimiento del syllabus y diseño instruccional. De la misma manera, se estructura en 16 semanas de estudio, cada semana con su respectivo recursos y actividades. Se evidencia que el $49.1 \%$ de los encuestados responden satisfactoriamente en función de la secuenciación coherente y adecuada.

En cuanto a las herramientas de comunicación e interacción utilizadas en la plataforma MOODLE UCSG, un $42,1 \%$ de los estudiantes contestan que fue un excelente recurso comunicacional. Seguido de un $30 \%$ que responden muy bueno. En este sentido se tiene un escenario favorable de un $70 \%$ aproximadamente que muestran su satisfacción a las herramientas de comunicación e interacción.

En lo que respecta al entorno de la plataforma, los estudiantes del aula de prueba consideran en un 51,7\% que es de fácil manejo, sumado a un $31,1 \%$ que también considera que la interfaz es buena. En este escenario se tiene que un $83 \%$ aproximadamente están conformes al entorno de la plataforma MOODLE UCSG.

Como pregunta de cierre del cuestionario, se establece el nivel de satisfacción de los estudiantes por el curso en modalidad virtual, como forma combinada de estructura curricular con las asignaturas de estudios generales. Los estudiantes encuestados manifiestan que el $45,6 \%$ se sienten motivados en el uso de la plataforma, sumado a un 31,5\% que la motivación es buena. Con este resultado se precisa que el resultado de la aplicación de la prueba es positivo.

Con base en el análisis realizado, el investigador expone que las TIC no solo trascienden en lo tecnológico con herramientas y programas; sino en la transferencia de conocimiento y que se ajusta a la necesidad de los sujetos en formación con bases en la pedagogía y la tecnología para diseñar recursos significativos para el desarrollo del proceso de enseñanza y aprendizaje. Se presentan como un grupo de herramientas que permiten la transmisión, el procesamiento y el almacenamiento de la información de manera digitalizada, y la creación de hardware y software para su utilización en la enseñanza (Medina, De la Herrán y Sánchez , 2012).

De acuerdo con Gros (2018), muchas plataformas en línea tienen soluciones integradas para facilitar el aprendizaje colaborativo con recursos comunicacionales como base de los aprendizajes emergentes (Castañeda \& Adell, 2013), que generan experiencias más atractivas y ha sido insumo para el desarrollo del aprendizaje en línea.

Por todo lo antes mencionado, y al seguir la línea de García Aretio, García Blanco y Ruiz (2017) acerca de las TIC, los cambios en la educación superior traen nuevos paradigmas que, al estar centrado en dos objetos (estudiante y docente), cambian el objetivo de los modelos pedagógicos y de sus dimensiones, basándose en la interacción participativa, constructiva y cooperativa del proceso de aprendizaje, desarrollando habilidades y destrezas en los estudiantes y potenciando el proceso de enseñanza-aprendizaje.

La IX Jornada de Futuro Español (2019) representantes del sector tecnológico y universitario de habla hispana analizan las TIC a favor de la educación y como alternativa para la sostenibilidad económica de los países que se basan en el capital humano para su crecimiento. En este encuentro, se precisa que la tecnología no es el futuro, sino el presente y que está transformando la capacidad de aprender (Futuro en Español, 2019). 
En efecto, dado los crecientes niveles de conectividad y acceso a la internet, el ciberespacio o web es fundamental para el desarrollo de la educación. Se justifica en la dinámica de sus recursos, herramientas y aplicaciones que sustentan la virtualización como una alternativa inmediata, masificadora y sinérgica que promueva la cultura de la innovación sin que este atada a circunstancias espaciales y burocráticas (Romero, Cuenca, \& Lahera, 2019).

\section{El paradigma de la enseñanza remota emergente}

El impacto de la COVID-19 en el sistema de educación mundial y ecuatoriano genera cambios radicales en los modelos pedagógicos ejecutados hasta marzo 2020. Entonces, debido a la emergencia sanitaria se migra de manera radical a la virtualidad, dando paso a este nuevo paradigma y de esta forma ofrecer una respuesta inmediata al proceso de enseñanza con el fin de evitar la paralización de un derecho fundamental como es la educación. A partir de ello, se originan los marcos normativos y legales, para garantizar la educación de calidad en este contexto.

Este nuevo escenario, cambia los hábitos y fortalece las herramientas tecnológicas que, entonces, se presentaban de manera tímida y sin mucha demanda. El confinamiento obliga a recurrir a las transformaciones pedagógicas, generando este paradigma educativo en donde el uso de las tecnologías se vuelve fundamental. En el contexto educativo, los espacios físicos se reemplazan por espacios virtuales con el uso de herramientas para videoconferencia como ZOOM, Google Meet, Microsoft Teams y demás; que se convierten en el medio necesario y obligatorio para la comunicación y transferencia de información (Aguilar, 2020).

Ese paso del aprendizaje en espacios presenciales a espacios virtuales, no se presenta como una alternativa sino como una necesidad que no solo se contextualiza en tiempos de pandemia, sino también en futuro inmediato donde el aprendizaje se desarrolla en espacios dinámicos que permitan el uso de tecnologías de manera eficiente, propiciando la conectividad de la comunidad educativa con recursos provistos desde las instituciones de educación superior (Crespo, 2021).

Considerando que los cambios en los modelos educativos establecen como centro del proceso formativo al estudiante, en la educación virtual se convierte en un protagonista indiscutible. La clase magistral por videoconferencia no es lo mismo que una clase magistral presencial, ya que en lo primero convergen acciones que se transforman en recursos en un proceso de orientación colaborativa y cooperativa que permiten al estudiante explorar contenidos en diferentes recursos educativos (Valero, Castillo, \& Rodríguez, 2020).

Los momentos de crisis son la motivación principal para innovar y, en ese contexto, tanto personas como empresas han trabajado para desarrollar tecnologías que permitan superar estas situaciones. Sin embargo, no solo se debe pensar como una medida alternativa sino más bien como el momento correcto para evidenciar que la educación requiere ese salto a lo moderno, a lo necesario y pertinente y que, en un momento de crisis mundial, un mecanismo que permita avanzar y evolucionar (Hurtado, 2020).

La influencia de estos autores en el desarrollo de las TIC y el fortalecimiento de la educación parten con el desarrollo de la tecnología, en un inicio se centran en el profesor para transmitir información directa al estudiante sin ningún tipo de interacción. Ante ello, se plantean definiciones conceptuales de virtualidad de la educación, interacción, colaboración las cuales cambian el paradigma educativo y sus tendencias lo cual, con apoyo de la tecnología emergente, se logran modelos dentro de la tecnología educativa con herramientas para el 
desarrollo de actividades (interactivo) y centrada en docentes y estudiantes (colaborativocooperativo).

Esto motiva a un cambio en la práctica educativa con la generación de espacios y ambientes virtuales en el cual, la interacción entre profesor y estudiante se da en la misma línea comunicacional. En la actualidad, virtualizar supone un modelo de gestión pedagógica que contribuye a la educación con nuevos escenarios de aprendizaje (Chan, 2016).

La tecnología siempre ha representado una herramienta que facilita la vida del ser humano. En términos más precisos, la tecnología es el resultado de diferentes teorías y concepciones que dan solución a problemas de la sociedad en función de tiempo, espacio y distancia, ya que transitan desde la idea y la concepción para transformarse en teoría y acción que respaldan la fundamentación científica de su estudio e implementación en el perfeccionamiento de la educación y su importante abordaje como camino al cambio y evolución de la sociedad.

\section{Conclusiones}

La importancia de la integración de la tecnología educativa con las asignaturas de estudios generales y su virtualización es el camino para la optimización de procesos educativos y formativos. Se basa en teorías y enfoques relacionados con el constructivismo y conectivismo que se estructuran en procesos de aprendizaje a través de recursos tecnológicos, que concurren a la interacción entre docente y estudiantes en un contexto innovador.

La virtualización de las asignaturas de estudios generales en la educación tiene un escenario viable ya que permite no solo la formación integral en función de los aspectos propios del desarrollo profesional, sino también un desarrollo de la habilidad tecnológica de los estudiantes y una alternativa para la optimización de los recursos, combinando modalidades de aprendizaje.

\section{Referencias}

Aguilar, F. (2020). Del aprendizaje en escenarios presenciales al aprendizaje virtual. Estudios Pedagógicos(3), 213 - 223. doi:10.4067/S0718-07052020000300213

Barreto, R., \& Iriarte, F. (2017). Las Tic en educación superior: Experiencias de innovación. Barranquilla: Universidad del Norte.

Buhalis, D., \& Deimezi, O. (2003). E-tourism developments in Greece. Information Communication Technologies adoption for the strategic management of the Greek tourism industry. Tourism and Hospiyality Research, 5(2), 103-127.

Castañeda, L., \& Adell, J. (2013). Entornos personales de aprendizaje: claves para el ecosistema educativo en red. . Alcoy: Editorial Marfil S.A.

Chan, M. (2016). La virtualización de la educación superior en América Latina: entre tendencias y paradigmas. Revista de Educación a Distancia, 1(48), 1-32. Obtenido de https:/www.um.es/ead/red/48/chan.pdf

Crespo, D. (2021). Enseñanza remota emergente. Polo de Conocimento, 6(6), 1040-1051. doi: $10.23857 /$ pc.v6i6.2802

EDUCASE. (2019). Horizon Report Preview. Obtenido de https://library.educause.edu/ /media/files/library/2019/2/2019horizonreportpreview.pdf 
Futuro en Español. (Octubre de 2019). IX Jornadas Futuro en Español. Obtenido de Círculo Logroñés, Rectorado UNIR Y Auditorio del Ayuntamiento de Logroño: http://www.xn--futuroenespaol1nb.es/2019-2/ix-jornadas-futuro-en-espanol/

Henández, R., Fernández, C., \& Baptista, M. (2014). Metodología de la Investigación. México: Mc Graw Hill.

Hurtado, F. (2020). La educación en tiempos de pandemia: Los desafíos de la escuela del siglo XXI. Revista Venezolana de Ciencia y Tecnología(44), 176-187. Obtenido de https://www.grupocieg.org/archivos_revista/Ed.44(176187)\%20Hurtado\%20Tavalera_articulo_id650.pdf

Medina, De la Herrán y Sánchez . (2012). Formación pedagógica y práctica del profesorado. Madrid: Centro de Estudios Ramón Areces S.A.

Medina, J., \& Rea, S. (2017). La educación a distancia como mecanismo de inclusión social en las Universidades del Ecuador. Alternativas, 2(17), 5 - 10. doi:https://doi.org/10.23878/alternativas.v17i2.113

Rodríguez, L. (2007). Probabilidad y estadística para ingenieros. Guayaquil, Ecuador: Escuela Superior Politécnica del Litoral.

Romero, R., Cuenca, Y., \& Lahera, M. (2019). Las Tecnologías de la Información y las Comunicaciones en el contexto de la actividad científica. Opuntia Brava, 11(2), 70 - 79. Obtenido de http://opuntiabrava.ult.edu.cu/index.php/opuntiabrava/article/view/742

UNESCO. (2009). Conferencia Mundial sobre la Educación Superior - 2009: La nueva dinámica de la educación superior y la investigación para el cambio social y el desarrollo. Obtenido de http://www.unesco.org/education/WCHE2009/comunicado_es.pdf

UNESCO. (2019). Las TIC en la Educación. Obtenido de https://es.unesco.org/themes/tic-educacion

Valero, N., Castillo, A., \& Rodríguez, N. (2020). Retos de la educación virtual en el proceso enseñanza aprendizaje durante la pandemia de Covid-19. Dominio de las Ciencias, 1201 - 1220. doi:https://doi.org/10.23857/pocaip 